\title{
Attractivité des territoires ruraux et choix de carrière dans l'enseignement vétérinaire
}

\section{Sylvain Dernat}

\section{(2) OpenEdition}

\section{Journals}

Édition électronique

URL : http://journals.openedition.org/cdg/1188

DOI : $10.4000 /$ cdg. 1188

ISSN : 2107-7266

Éditeur

UMR 245 - CESSMA

Référence électronique

Sylvain Dernat, «Attractivité des territoires ruraux et choix de carrière dans l'enseignement vétérinaire », Carnets de géographes [En ligne], 10 | 2017, mis en ligne le 30 septembre 2017, consulté le 24 septembre 2020. URL : http://journals.openedition.org/cdg/1188; DOI : https://doi.org/10.4000/cdg 1188

Ce document a été généré automatiquement le 24 septembre 2020.

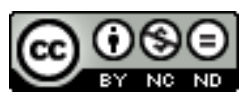

La revue Carnets de géographes est mise à disposition selon les termes de la Licence Creative Commons Attribution - Pas d'Utilisation Commerciale - Pas de Modification 4.0 International. 


\title{
Attractivité des territoires ruraux et choix de carrière dans l'enseignement vétérinaire
}

\author{
Sylvain Dernat
}

1 L'objectif de cette thèse était de contribuer à la compréhension de ce qui fonde, aujourd'hui en France, le choix de la spécialisation des étudiants des écoles vétérinaires et, en particulier, vis-à-vis de la désaffection qui touche la filière rurale. En effet, la crise de vocation que cette dernière traverse depuis de nombreuses années provoque une baisse significative du nombre de praticiens et les prévisions pour le futur sont plus préoccupantes encore. Or, la présence de vétérinaires en zones rurales est porteuse d'importants enjeux sociétaux : économiques, sanitaires ou sociaux. Les différentes politiques publiques incitatives mises en place se sont avérées, jusqu'ici, peu capables de contrer ce phénomène. De même, la seule prise en compte des caractéristiques socio-démographiques des étudiants, proposée par les pouvoirs publics pour expliquer leurs choix de carrière au sein du cursus vétérinaire, semble insuffisante. En effet, ces choix semblent provenir plutôt d'aspirations nouvelles en termes d'organisation de l'activité professionnelle, mais également de vie personnelle. Ils pourraient ainsi être corrélés à une caractéristique de la désertification vétérinaire rurale : elle n'est pas homogène dans l'espace français. Certains territoires attirent toujours des praticiens, alors que d'autres semblent négligés.

2 La recherche présentée ici a donc analysé le cursus vétérinaire dans cette optique en questionnant les choix de carrière des étudiants dans une dimension psychosociale au regard de sa dimension spatiale. Pour cela, la théorie des représentations sociales a été mobilisée. Ce décalage du focus, des caractéristiques de la population étudiante vers la prise en compte psychosociale de leurs représentations, a ainsi offert deux finalités à cette recherche. L'une, heuristique, a visé la production de connaissances sur les processus de choix de carrière. L'autre, pragmatique, a tenté d'élaborer des propositions de solutions pédagogiques d'accompagnement de ces choix. 
3 Dans un premier temps, la finalité heuristique peut être questionnée. Ici, les travaux proposés ont mis en évidence l'intérêt de l'approche représentationnelle pour comprendre les choix de carrière des étudiants. Dans ce sens, l'analyse systémique des représentations mettant en rapport la représentation socio-professionnelle du vétérinaire rural avec la représentation socio-spatiale des territoires ruraux semble être une perspective intéressante. La recherche a ainsi montré le lien fort qui existe entre les aspects professionnels des choix de carrière et les aspects spatiaux (isolement, accessibilité...). Il a été possible également d'aller au-delà de ces aspects en montrant que les éléments spatiaux mettent en évidence des aspirations plus individuelles (fonder une famille, besoin de s'épanouir...). À un niveau théorique, la modélisation utilisant un double projet représentationnel (Figure 1), ou double "Toblerone », a permis l'inscription de ce système de représentations dans une optique temporelle. Elle fait appel, à un instant $t$, à la fois au passé de l'étudiant et de ses groupes sociaux, et également à ses projections dans le futur.

4 De plus, les travaux de cette thèse ont également permis de questionner la possibilité de transformation des représentations dans un contexte pédagogique, au travers de la mobilisation du concept de conflit socio-cognitif. Cela a été réalisé par la mise en place d'un dispositif collaboratif spécifique : le jeu de territoire. Ici, les résultats ont permis de montrer que le conflit socio-cognitif produit permet d'introduire le changement des représentations et de favoriser la prise en compte du territoire rural dans l'analyse de l'activité du vétérinaire par les étudiants. Toutefois, celui-ci ne va pas de soi. Il dépend de l'animation du dispositif, notamment de la gestion pédagogique du statut des différents participants, et du vécu des étudiants. Cependant, lorsqu'il fonctionne, le jeu de territoire pourrait favoriser la prise en compte des éléments relatifs au territoire d'activité dans leur parcours d'orientation par la médiation de cartes co-construites (Figure 2).

5 Les étudiants vivent donc une transition qu'il faut accompagner en créant un espace social de médiation dans le cursus entre, d'une part, le monde professionnel et les territoires de pratique, et d'autre part les systèmes de représentations. Cela rejoint alors l'aspect pragmatique du questionnement de cette thèse. En effet, l'utilisation de critères socio-démographiques pour approcher les choix des étudiants vétérinaires visà-vis de la rurale semble réductrice. La féminisation, l'origine urbaine ou de catégories socio-professionnelles supérieures apparaissent comme des indicateurs mais n'expliquent que peu les parcours des étudiants. La signification donnée au territoire d'activité est plus prometteuse: elle montre l'imbrication de plusieurs niveaux de réflexion des étudiants.

6 Toutefois, les résultats de cette thèse suggèrent qu'il faut sortir d'une perspective académique classique, disciplinaire et tendant aux seuls savoirs cliniques dans l'enseignement vétérinaire. Les propositions pédagogiques formulées, à l'image du jeu de territoire, prennent forme dans un modèle permettant une ouverture vers des savoirs non-académiques, utiles à la formation de futurs professionnels. Ces savoirs, notamment de connaissance de l'espace d'activité du vétérinaire, favorisent alors l'accumulation par les étudiants d'un capital spatial propice à mieux les intégrer dans une possible carrière en pratique rurale. Ils ne demandent pas de repenser le curriculum mais simplement de l'enrichir, et apparaissent comme une opportunité de développement réalisable. Ainsi, au- delà du travail visant à comprendre les liens qui unissent les choix de carrière des futurs vétérinaires avec la dimension spatiale de ces 
choix, cette thèse propose donc des clés pédagogiques potentiellement actionnables pour favoriser une installation des jeunes praticiens: la prise en compte de leur transition vers le monde professionnel et l'articulation de savoirs leur permettant de mieux s'y insérer. Il serait donc possible d'envisager que les résultats de cette recherche puissent être également transposés dans d'autres formations liées à des professions concernées par la désertification des territoires ruraux, notamment celle des médecins. Même si ces formations ont leurs propres spécificités, une partie du traitement des difficultés auxquelles elles sont confrontées pourrait passer par l'accompagnement pédagogique des transitions socio-professionnelles, notamment par une analyse mêlant représentations sociales et territoires.

Figure 1. Modélisation théorique du réseau de représentations.

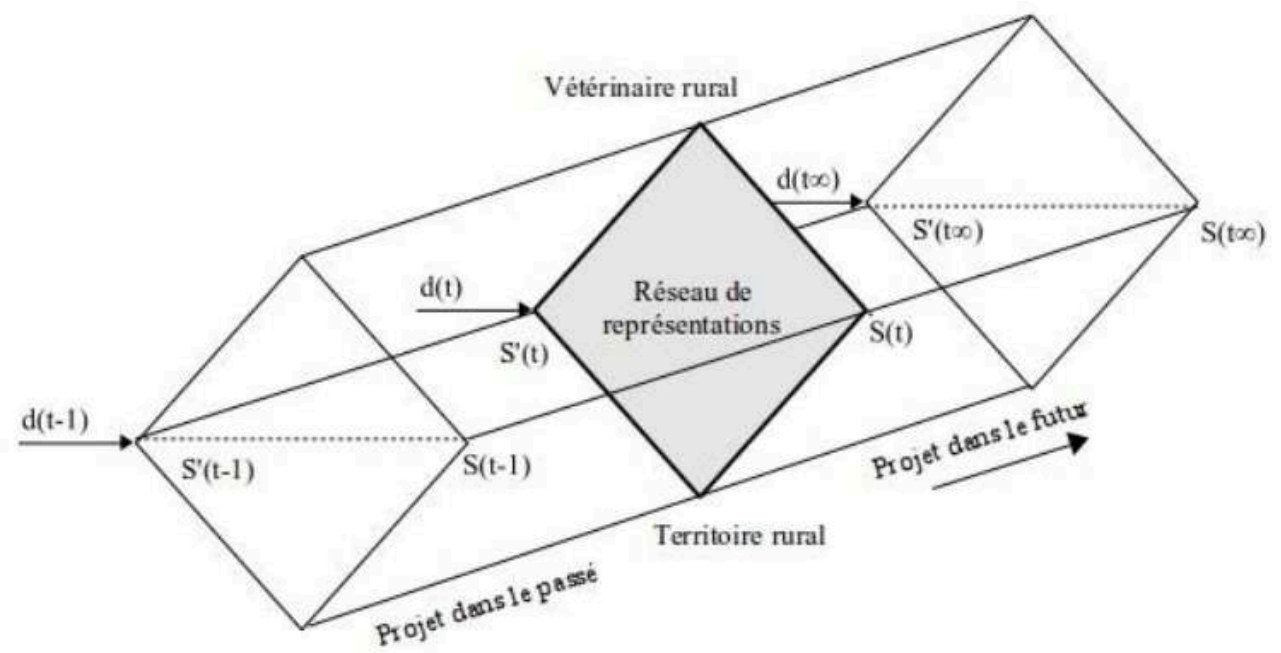


Figure 2. Le jeu de territoire - Utilisation de la médiation par les cartes co-construites.

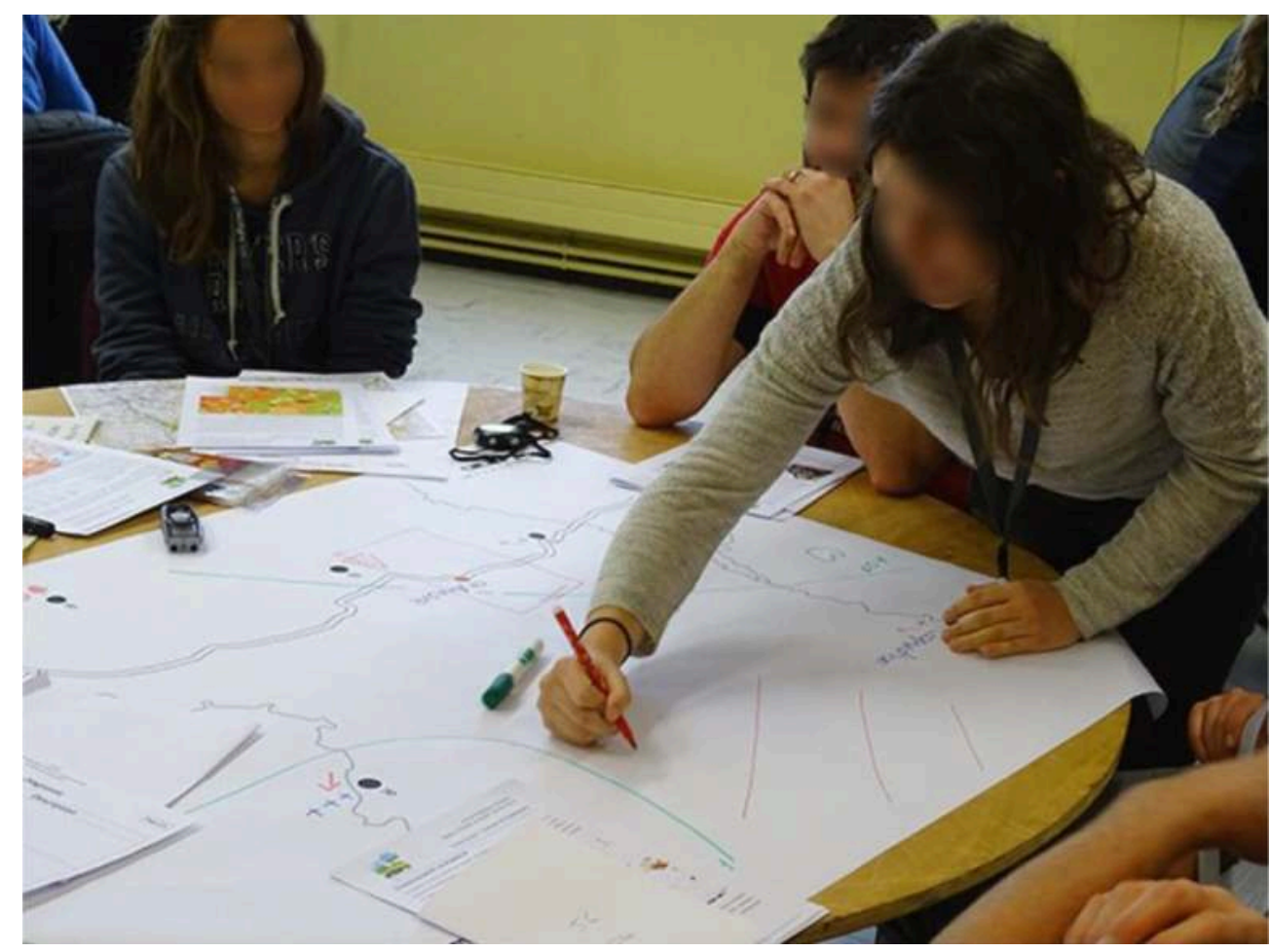

\section{Discipline}

Géographie sociale, psychologie sociale et sciences de l'éducation.

\section{Directeurs}

Sylvie Lardon, Directrice de recherche en géographie, INRA Clermont-Ferrand Arnaud Siméone, Maître de conférences en sciences de l'éducation, Université Lumière Lyon 2

\section{Université}

Université Clermont-Auvergne

Membres du jury de thèse, soutenue le 4 avril 2016

Jérôme Eneau, Professeur, Université de Rennes 2, examinateur Nikos Kalampalikis, Professeur, Université Lumière de Lyon, examinateur Sylvie Lardon, Directrice de recherche, INRA Clermont-Ferrand, encadrante Michel Lussault, Professeur, École normale supérieure de Lyon, examinateur Christine Mias, Professeur, Université Jean Jaurès de Toulouse, rapporteur Thierry Ramadier, Directeur de recherche, CNRS Strasbourg, rapporteur Arnaud Siméone, Maître de conférences, Université Lumière de Lyon, encadrant

\section{Situation professionnelle à l'issue de la thèse}

Assistant ingénieur à l'INRA, UMR Territoires

Enseignant vacataire à VetAgro Sup.

\section{Courriel}

sylvain.dernat@inra.fr 
INDEX

Thèmes : Carnets de soutenances 